\title{
Wiedererfahrung durch Psychotherapie modifiziert Geist und Gehirn*
}

\author{
Maggie Schauer Thomas Elbert Silke Gotthardt Brigitte Rockstroh Michael Odenwald \\ Frank Neuner
}

Klinische Psychologie, Universität Konstanz, und vivo e.V., Konstanz, Deutschland

\author{
Schlüsselwörter \\ Posttraumatische Belastungsstörung · Gehirn · Narrative \\ Expositionstherapie · Neuropsychotherapie
}

\section{Zusammenfassung}

Psychotherapie verändert klinische Symptome und mentale Funktionen und damit auch Leistungen des Gehirns. Dies bedeutet, dass sich die Architektur des Gehirns durch erfolgreiche therapeutische Intervention modifizieren lässt. Tatsächlich kann der Nachweis sogar auf makroskopischer Ebene gelingen, wie wir hier am Beispiel der Behandlung seelischer Erkrankungen in Folge von traumatischem Stress darlegen. Die Narrative Expositionstherapie (NET) wurde dabei mit Standardbehandlungen (TU; treatment as usual) bei traumatisierten Asylsuchenden in Deutschland verglichen. Der Erfolg der NET spiegelte sich nicht nur in den Symptomkennwerten wider, sondern auch in neuromagnetischen Messungen. Diese zeigten in der 6-Monats-Katamnese, dass sich die Hirnaktivität in der NET-Gruppe an diejenige einer psychisch unauffälligen Normstichprobe angenähert hatte. In der TU-Gruppe war dies nicht der Fall. Neurophysiologische Messungen können somit (1) den Therapieerfolg validieren, (2) Wirkmechanismen einer Therapieform überprüfen, wodurch ein Zugang zu besserer Modellierung geschaffen wird, (3) die differentialdiagnostischen Möglichkeiten erweitern und (4) uns einmal mehr lehren, dass Geist und Gehirn lediglich zwei Informationsebenen ein und desselben Phänomens darstellen.

\section{Key Words}

Posttraumatic Stress Disorder · Brain - Narrative

Exposure Therapy · Neuropsychotherapy

\section{Summary}

Imaginary Reliving in Psychotherapy Modifies Mind and Brain

The psychotherapeutic process modifies clinical symptoms in conjunction with mental functioning, i.e., it includes alterations of brain performance. This leads to the conclusion that successful psychotherapeutic interventions may modify the architecture of the brain. Imaging of corresponding changes may indeed be possible on a macroscopic level, as we have shown regarding the treatment of mental disorders due to repeated experiences of traumatic stress. In a controlled trial, narrative exposure therapy (NET) was compared to treatment as usual (TU) of traumatised asylum seekers in Germany. The success of NET not only showed up in symptom scores but also in parameters of neuromagnetic activity. During the 6-month follow-up brain activity in the NET group but not in the TU group became more similar to that of normal controls. We conclude that neurophysiological measurements (1) may validate the success of the therapy, (2) allow testing of causative models of the therapy, (3) enhance the range of differential diagnostic measurements and (4) indicate once more that mind and brain are merely different channels of information of one and the same phenomenon. 


\section{Einleitung}

Griesingers um die Wende zum 20. Jahrhundert geprägtes «Geisteskrankheiten sind Gehirnkrankheiten» wird fast schon als triviales Dogma des Zeitgeists gehandelt. Gleichzeitig ist die Medizin und überwiegend wohl auch noch die Psychologie vom cartesianischen Dualismus durchdrungen, der Seele und Geist als nicht von den «Mächten der Materie» herleitbar begreift. In der Tat entwickelte sich die Kognitive Psychologie und in der Folge auch die Klinische Psychologie weitgehend als von der Biologie losgelöstes Feld, das sich letztlich auf eine Art Computermetapher verließ [z.B. Neisser, 1967]. Bevor wir argumentieren, dass Geist und Gehirn nur zwei verschiedene Beschreibungsebenen ein und desselben menschlichen Wesens darstellen, wollen wir die Computermetapher noch einmal kurz in Erinnerung rufen:

Wer erinnert sich nicht mehr oder weniger schmerzlich an einen Absturz des Textverarbeitungsprogramms auf dem Rechner? Kaum jemand käme deswegen auf die Idee, den Rechner zur Reparatur zu geben - eben ein Softwareproblem. Könnte es also sein, dass die neurobiologisch basierte Fehlersuche etwa bei Schizophrenie oder posttraumatischer Belastungsstörung (PTSD) genauso sinnlos ist wie die Überprüfung von Chips und Festplatten im Rechner, wenn uns ein Softwarebug ärgert? Natürlich ist auch das Softwareproblem nicht ohne materielle Grundlage. Die Bits in Form von Magnetisierungen auf der Festplatte sind eben in falscher Ordnung. Die Funktionstüchtigkeit von Speichern und Festplatte an sich mag dabei einwandfrei sein, sie müssten nur neu formatiert und beschrieben werden. Durchaus denkbar wäre entsprechend im Gehirn, dass die Verbindungen zwischen Neuronen, also etwa synaptische Gewichte, ähnlich den Bits auf der Festplatte, falsch justiert sind, der zugrunde liegende Apparat aber, in diesem Fall die Justierbarkeit von synaptischen Verbindungen, sehr wohl in Ordnung ist. Neuroimaging mit dem Versuch, die durch seelische Erkrankung bedingte Funktionseinschränkung zu präzisieren, also quasi den Zustand des Rechners nach «Absturz eines Programms» (nicht des ganzen Betriebssystems), bliebe dann sinnlos, auf jeden Fall aber weit davon entfernt, ätiologischem Verständnis und damit letztlich auch therapeutischem Erfindungsreichtum weiterzuhelfen.

Bei genauerer Betrachtung aber verbirgt sich hinter der Computermetapher doch nichts anderes als der Irrtum Descartes', die fundamentale Eigenschaft der Materie verkennend, mit sich in Wechselwirkung zu treten und somit «Verhalten» zu zeigen. Im realen Gehirn modifiziert die Funktion des Geistes, also quasi die Software, unablässig die funktionelle und strukturelle Organisation des Gehirns, also quasi die Hardware. Erfahrungen beeinflussen Hirnfunktion und -struktur bis hin $\mathrm{zu}$ makroskopisch messbaren materiellen Veränderungen [z.B. Elbert et al., 2006; Elbert und Rockstroh, 2004]. Diese nun modifizierte Hirnorganisation beeinflusst fortan alle weitere Verarbeitung externer und interner Reize, also psychische Funktionen. Da die interne Reizverarbeitung den Löwenan- teil der Prozessierung bildet (über neun Zehntel des Einstroms an kortikalen Synapsen etwa stammen aus internen Schaltkreisen), sind lebende Organismen sich selbst beständig reorganisierende dynamische Systeme. Dabei reorganisieren sich nicht nur synaptische Gewichte, sondern es verändern sich auch Genexpression, Verbindungen, Datenströme und damit Verarbeitungsstrukturen und Funktionssysteme.

Um diese Prozesse zu erläutern, fassen wir zunächst einige Erkenntnisse aus der Forschung zur Neuroplastizität zusammen. Darauf folgend stellen wir am Beispiel seelischer Erkrankungen in Folge von psychischem Trauma dar, dass eben diese Erfahrungen schrittweise das Geist-Gehirn-System verändern und somit ein Spektrum an Symptomen nach sich ziehen können. Da wie gesagt (in diesem Fall traumatische) Erfahrungen das Gehirn in seiner Funktionsweise verändern, lassen sich, anders als bei somatischen Erkrankungen, klare Störungsbilder nicht voneinander abgrenzen. Der Versuch der Kategorisierung etwa nach Achse-I-Störungen muss dann in der Regel mit der häufigen Diagnose komorbider Erkrankungen bezahlt werden.

Erkrankungen mit Symptomen des Traumaspektrums eignen sich hervorragend für diese Diskussion, da in diesem Bereich ätiologische Modelle und effektive Psychotherapie vergleichsweise weit entwickelt sind [Schauer et al., 2005]. Tatsächlich werden die letzten Abschnitte dieses Beitrags zeigen, dass PTSD und Depression nicht nur im Bereich des Verhaltens und Empfindens, sondern auch auf der Ebene der Gehirnveränderungen beschrieben werden müssen. Auf keine dieser Informationsebenen dürfen gute Wissenschaft und gute Praxis verzichten. Schließlich sei an diesem Beispiel demonstriert, dass Psychotherapie makroskopische Veränderungen im Gehirn induzieren kann und dass sich aus deren Messungen wiederum Modelle möglicher Wirkmechanismen eingrenzen lassen.

\section{Neuroplastizität}

Erfahrungen modifizieren nach der Hebb'schen Regel beständig die synaptischen Gewichte im Gehirn über LTP (longterm potentiation) und LTD (long-term depression). Feuert erst das prä- und kurz darauf das postsynaptische Neuron, so werden die Verbindungen verstärkt, d.h. das präsynaptische Neuron erhält mehr Macht, das postsynaptische Neuron zu depolarisieren. Im umgekehrten Fall oder bei asynchroner Feuerung kommt es zur Abschwächung der Konnektion. In der Folge verändern sich die Synapsen morphologisch, neue Spines werden gebildet, und es kann zu Dendriten- und Axonwachstum kommen, was vermehrte gliale Unterstützung und letztlich Angiogenese anfordert. Tatsächlich belegen Studien des repräsentationalen Kortex, dass die Rindenfelder sich in Abhängigkeit von verhaltensrelevanter Erfahrung kontinuierlich und in der Art massiv verändern, dass sowohl funktionelle als auch strukturelle Veränderungen in den Repräsentationsarealen makroskopisch messbar werden [Elbert und Bonhoeffer, 2002; Elbert und Rockstroh, 2004]. So konnten 
wir mittels magnetischer Quelllokalisation zeigen, dass bei Personen mit extensiven Fingerübungen, wie etwa bei Musikern, das Rindenfeld, das die Finger repräsentiert, vergrößert ist [Elbert et al., 1995]. Strukturelles MRI (Magnetic Resonance Imaging) belegt, dass sich der entsprechende Bereich der Zentralfurche im Millimeterbereich, also makroskopisch, in Folge von Übung vertieft [z.B. Gaser und Schlaug, 2003]. Während «kritischer Phasen» in der Entwicklung erfährt Neuroplastizität eine spezielle Ausprägung, bei der bereits sensorische Reize ohne explizite Verhaltensrelevanz ausreichen, um die Organisation sensorischer Rindenfelder zu verändern [z.B. Bao et al., 2003]. Demgegenüber erfordert funktionelle Reorganisation im adulten Kortex, dass die sie auslösenden Reize an Verstärker gekoppelt sind, also einen verhaltensrelevanten Kontext [Elbert und Rockstroh, 2004]. Die Wirkung dieser Verstärker wird über cholinerge [Kilgard und Merzenich, 1998] und dopaminerge Bahnen [Bao et al., 2001] vermittelt, die ihrerseits selbst plastischer Modifikation unterliegen und sich zu maladaptiver Organisation ausprägen können, wie etwa bei Psychosen, deren Kern in der Verstellung der Verstärkersysteme liegt.

Die Studien von Levine [1957, 2002], Meaney [2001] und Kollegen belegen eindrücklich die beachtliche Prägung und Modifikation der Hirnstruktur durch elterliche Einflüsse und Erfahrungen während der Entwicklung und demonstrieren die Macht sozialer Interaktion, Genexpression und damit neuronales Substrat nachhaltig zu modellieren. Daraus ergeben sich in der Folge verändertes Verhalten, veränderte Persönlichkeit und letztlich massive Einflüsse auf seelische wie somatische Gesundheit während des späteren Lebens [Hellhammer, 2005]. Solche plastischen Modifikationen sind nicht notwendigerweise adaptiv. Stressreiche Lebensereignisse können insbesondere in kritischen Perioden die Organisation von Gehirn und Geist auch beeinträchtigen. Kindheit und Jugend sind kritische Perioden für die kognitive und emotionale Entwicklung [z.B. Steinberg, 2005] und bilden vulnerable Phasen für die Entwicklung der Reaktionen unter Stress bzw. allostatischer Belastung [Charmandari et al., 2003]. Bereits mehrere traumatische Erlebnisse sind in der Lage, eine Kaskade von dynamischen Veränderungen, messbar in Geist wie Gehirn, nach sich zu ziehen, die dann in einer erhöhten Vulnerabilität für weitere Stressoren resultiert. Hinreichend viele traumatische Erlebnisse führen immer in die Erkrankung, wenngleich es manches Mal mehr als zwei Dutzend schlimmer Erlebnisse von Angst und Hilflosigkeit bedarf, bis es zur Ausprägung einer PTSD kommt [Schauer et al., 2003; Neuner et al., 2004a].

\section{Traumatische Erfahrungen verändern Struktur und Funktion von Geist wie Gehirn}

\section{Beispiel Gedächtnis}

Symptome des Traumaspektrums lassen sich als Folge plastischer Veränderungen aufgrund der traumatischen Erfahrun- gen begreifen. Normalerweise werden wesentliche Erfahrungen detailliert und in chronologischer Abfolge im episodischen Gedächtnis kodiert. Diese autobiographischen kontextuellen Speicherinhalte, auch als «cold memory» bezeichnet [Metcalve und Jacobs, 1996], beantworten dann die «Journalistenfragen»: Was? Wann? Wo? Wer? Warum? Kaltes Gedächtnis beinhaltet dieses Wissen und bettet spezifische Erfahrungen in Lebenszeitperioden ein.

Die sensorisch-perzeptuellen Elemente zusammen mit den emotionalen Inhalten bilden das «heiße» Gedächtnis. Nichtdeklarativ und implizit sind dessen Inhalte einem semantischen Zugriff nur über den Umweg der Imagination zugänglich, können dann aber auch (wieder) in Worte gefasst werden. Das heiße Gedächtnis beinhaltet szenische Bilder, Gerüche, Schreie, Lachen, aber eben auch die damit verknüpften Gefühle. Der Abruf von Inhalten, etwa der Geburtstagsfeier, des ersten romantischen Kusses, dem Autounfall, dem Überfall usw. aktiviert daher immer auch Emotionen, d.h. Reaktionsdispositionen und damit in unterschiedlicher Form die Stressachsen. Normalerweise sind die Inhalte des kalten Gedächtnisses (z.B. es war im Frühling 2003, an einem sonnigen Tag, etwa um 11 Uhr morgens, als ich zum ersten Mal auf einem Pferd galoppierte) mit heißen Gedächtnisinhalten verknüpft (z.B. ich spürte den Wind auf meinen erhitzten Wangen und konnte das Sägemehl in der Reithalle riechen, so wie jetzt, wenn ich davon erzähle(!)); kognitive (z.B. «ich kann es!»), emotionale (z.B. freudige Erregung) und physiologische Elemente (z.B. Herzklopfen, Schwitzen) usw. werden orchestriert aktiviert. Bei Personen jedoch, die traumatische, also bedrohende Erlebnisse, begleitet von stärkstem Erleben von Angst, Horror oder Hilflosigkeit erfahren mussten, bricht später oder früher die Verbindung eines immer größer werdenden Netzwerks aus heißen Gedächtniselementen vom autobiographischen, deklarativen Gedächtnis ab. Zu welchem der traumatischen Erlebnisse dann die auf die Brust drückende Angst, der chlorhaltige Geruch oder der maskierte Folterer gehören, ist dem Opfer dann nicht mehr unmittelbar verständlich. Das Furchtnetzwerk [Lang, 1979] stabilisiert sich in Hebb'scher Eigendynamik; es lässt Koppelungen zum kalten Gedächtnis verblassen. Die Verbindung zum kalten Gedächtnis, zur eigenen Lebensgeschichte ist abgebrochen. Eine ähnliche Reizkonstellation wie damals kann deshalb nicht nur Erinnerungen wachrufen, wie das auch bei gesunden Personen für einen kurzen Moment der Fall wäre, sondern aktiviert das gesamte Furchtnetzwerk - welches sich während der Phase höchster physiologischer Erregung während der traumatisierenden Zeit gebildet hat - in der Gegenwart. Häufig geschieht dies unbewusst durch gleichzeitige Aktivierung mehrerer Elemente, die sich dann gegenseitig «zündend» verstärken. Begegnet ein Überlebender des Krieges Jahre später bei seinem schnellen Gang zum Arzt einem Verkehrspolizisten, so können die physiologische Erregung, die Sorgen um seine Gesundheit und der Anblick der Uniform das Furchtnetz- 
werk so stark aktivieren, dass er sich in den Krieg zurückversetzt fühlt, also einen Flashback erleidet. Traumatisierte können die Zuordnung ihrer Erinnerungen in den autobiographischen Kontext nicht ohne weiteres und meist nicht ohne psychotherapeutische Unterstützung leisten.

\section{Beispiel oszillatorische Hirnaktivität}

Wie können wir die Spuren des Furchtnetzwerks im Gehirn sichtbar machen? Natürlich in Assoziationsexperimenten, die entsprechende Verknüpfungen belegen können [Balliel et al., 2006], aber überraschenderweise auch einfach durch rasche Abfolge emotionaler Reize. Junghöfer et al. [2001, 2003] untersuchten den Fluss der Gehirnaktivierung auf die rasche Abfolge von emotional erregenden Bildern. Mit der gleichen Technik haben wir gezeigt, dass sich durch kontinuierlichen Einstrom erregender Reize das Furchtnetzwerk bei schwer traumatisierten Personen selbst dann aktivieren lässt, wenn die Reize keinen direkten Bezug zu den traumatischen Erlebnissen haben [Junghöfer et al., 2003; Elbert et al., 2006]. Die Schwelle zur Auslösung von Flashbacks ist dann erniedrigt. Während der sensorische Kortex sich bei traumatisierten wie nichttraumatisierten Personen durch schnelle Reizabfolgen deutlich aktivieren lässt, zeigen PTSD-Patienten zusätzlich Aktivierungen ihrer exekutiven und emotionalen Systeme im prä- und orbitofrontalen sowie im anterioren Gyrus cinguli (ACC) [Junghöfer et al., 2003].

Aber auch die Rhythmen und Strukturen verstellen sich im traumatisierten Gehirn. Während wir für Zusammenfassungen struktureller Veränderungen auf Elbert et al. [2006] und Kolassa und Elbert [2006] verweisen, wollen wir die Veränderungen in den rhythmischen Mustern näher darlegen, da sich zeigen lässt, dass sich diese durch psychotherapeutische Interventionen verändern lassen.

Langsame hochamplitudige Wellen mit fokalen Generatoren treten im Wachzustand gesunder Personen eher selten auf. Bei neurologischen wie psychiatrischen Erkrankungen sind solche abnormal langsame Generatoren dagegen eher die Regel und weisen auf pathologische Substrate hin [Rockstroh et al. 2001]. Die räumliche Verteilung und damit die Orte veränderter neuronaler Eigenschaften lassen sich über Magnetenzephalographie(MEG)-Messungen präzisieren. PTSD-Patienten weisen solche Regionen vermehrter langsamer abnormaler Generatoren vor allem präfrontal und linkstemporal, aber auch im ACC auf. Bei 23 Überlebenden von schwerer Folter, die alle auch die Kriterien einer PTSD erfüllten, beobachteten wir die deutlichsten Korrelationen $(r=0,4-0,65)$ in linksfrontalen und temporalen Regionen mit der Zahl traumatischer Erlebnisse und der Schwere klinischer Symptome, mit Dissoziation auch über die Kernsymptomatik der PTSD hinausreichend [Ray et al., 2006]. Diese Regionen dienen Sprache und exekutiven Funktionen, aber auch verbaler Enkodierung und Wiederabruf [Iidaka et al., 2000]. Dies kann erklären, warum insbesondere dissoziativen, aber auch traumatisierten Personen der deklarative, verbale Zugang zu den traumatischen Erlebnissen versperrt bleibt. Die Qualität heißen Gedächtnisses während der als erneuter Erfahrung empfundenen Erinnerung ist eben emotionaler und sensorischer Natur, ohne simultane sprachliche Expressionsmöglichkeit «sprachloser Terror».

Aus diesen Überlegungen heraus haben wir die Narrative Expositionstherapie (NET) entwickelt [Neuner et al., 2002, 2004b; Schauer et al., 2005]. Die folgende Untersuchung schildert, dass die NET einerseits klinische Symptome auch in schweren Fällen reduziert und andererseits tatsächlich die lokalen Grundrhythmen in betroffenen Hirnstrukturen zu verändern vermag, d.h. dass über rein psychotherapeutische Intervention das neuronale Substrat gezielt lokal, aber makroskopisch veränderbar ist. Die nur durch neurophysiologische Messungen zu beantwortende Frage dabei ist, ob die Psychotherapie eher normalisierend auf Hirnfunktion und -struktur wirkt oder ob diese Parameter eher zusätzliche Abweichungen von der Norm anzeigen. Letzteres würde man vermuten, wenn erfolgreiche Therapie das Furchtnetzwerk hemmt, aber nicht auflöst. Eine Reduktion der Verbindungen im Furchtnetzwerk - etwa über Mechanismen der LTD würde dagegen eher eine Normalisierung der Indikatoren der Hirnarchitektur erwarten lassen.

\section{Grundprinzipien der Narrativen Expositionstherapie}

Um Menschen nach entsetzlichen, lebensbedrohlichen Ereignissen zu behandeln, muss man verstehen, dass Traumatisierte nicht wieder in der Gegenwart angekommen sind. Die Bedrohung ist nicht vorbei. Die Wirklichkeit ist vielmehr das Trauma. Das eigentliche Hier und Jetzt erleben Traumatisierte als nur trügerische Realität. Durch die spezifische Gedächtnisproblematik nämlich kommt es zum Fehlen der Verortung (Raum) und Vergeschichtlichung (Zeit) der traumatischen Szene. Ihre Seele, ihr Gehirn ist gefangen im gegenwärtigen Schrecken: «Ich bin erfüllt von einer tiefen Beklemmung, die deutliche Empfindung einer drohenden Gefahr. Ich weiß es, und ich wusste es immer: Ich befinde mich ... im [Trauma]. Nichts ist wirklich, außer dem [Trauma]. Alles andere waren kurze Ferien, Sinnestäuschungen, ein Traum.» (Primo Levi: «Atempause»).

Um eine raum-zeitliche Rückbindung der traumatischen Erlebnisse zu erreichen, positioniert sich der Patient zusammen mit dem Therapeuten endlich außerhalb des Traumas (Exposition), nämlich in der Gegenwart, indem er beginnt, sein Trauma zu erzählen und sich gleichzeitig durch Wiedererleben des Vergangenen «mit allen Sinnen» intensiv in es hineinversetzt. Auf diese Weise sollen die sensorischen, emotionalen und kognitiven Erinnerungen an das Erlebnis verknüpft werden. Der Überlebende versetzt sich also emotional-imaginativ und unter Umständen auch in der Körperhaltung noch einmal intensiv in die traumatische Szene. Dieses Mal aber in Begleitung des Therapeuten und ohne den Kontakt zur Gegenwart 
zu verlieren. Nach einigen Minuten des Gehaltenwerdens in gleichzeitigem Erleben und Erzählen werden nach und nach alle Elemente des Erlebens der traumatischen Szene aus der Vergangenheit aktiviert, es aktualisiert sich die Erfahrung auf allen Ebenen in der Gegenwart (Gedanken, Gefühle, Bedeutungen, Empfindungen, Körperhaltung usw.) und doch im Schutz des «Sprechens über» in der Distanz zum «Damals»; so lange, bis das Erlebte sich autobiographisch ein-ordnen, benennen, be-greifen, verorten lässt und Erleichterung eintritt durch Habituation und Integration.

Nach und nach erstellt der Patient in den Behandlungssitzungen mit Unterstützung des Therapeuten eine detaillierte und konsistente Narration seiner Lebensereignisse und schließlich der gesamten Biographie in chronologischer Reihenfolge. Diese Autobiographie wird schriftlich festgehalten und bei jedem weiteren Lesen in den nachfolgenden Therapiesitzungen angepasst. Dabei liegt der Fokus auf der Einbettung der anfänglichen Fragmente der traumatischen Erinnerungen in ein sinnvolles Ganzes. Der Überlebende wird durch empathische Verbalisierung ermutigt, während der Erzählung seine damaligen Gefühle in der Gegenwart wiederzuerleben. Emotionale Verarbeitung schließt die Modifikation des ursprünglichen Furchtnetzwerkes ein. Sie ermöglicht die Rekonstruktion der autobiographischen Repräsentation des Erlebten. Menschen scheinen natürlicherweise zu versuchen, sich selbst zu heilen, indem sie ihre Erfahrungen erzählen. In vielen Fällen hilft der Prozess, Kontextinformationen, also «kalte» Gedächtnisinhalte aufzubauen, dabei, die «heißen» einzuordnen; die Menschen lernen, zwischen vergangener und gegenwärtiger Bedrohung zu unterscheiden. Da das Denken an das traumatische Erlebnis aber schmerzvolle Emotionen hervorruft, vermeiden die Überlebenden diesen Prozess oder kürzen ihn ab und versuchen, die Erinnerung so schnell wie möglich zu beenden. Dabei bleibt die Erfahrung aus, dass die Angst in Bezug auf das Erlebnis nicht ins Unermessliche steigt. Angst vor der Angst etabliert sich im Furchtnetzwerk, die PTSD chronifiziert. Die imaginative «Wiedererfahrung» ist das stärkste Mittel, um das Furchtnetzwerk «niederzureißen», da der Patient lernt, dass sensorische und emotionale Erinnerungen auch ohne vergleichbare gegenwärtige physiologische und affektive Erregung aktiviert werden können. Die Aufgabe des Therapeuten ist es, den Patienten bei der Organisation der Traumaerinnerungen - auch der schmerzhaften - zu unterstützen, vor allem bei der Verortung der Ereignisse in Raum und Zeit.

Es kommt auf diesem Wege zudem zu einer Gesamtschau des eigenen Lebens, zu einem organischen Erkennen von LebensMustern und Zusammenhängen, zu einer Würdigung der personalen Identität und der Biographie des Überlebenden. In der letzten Sitzung bekommt der Patient einen schriftlichen Bericht seiner Biographie, welcher vom Überlebenden und allen Zeugen (Therapeut, Dolmetscher, evtl. Beisitzer) unterzeichnet wird. Dies bildet gleichzeitig einen rituellen Abschluss des Prozesses.

\section{Modifikationen durch Psychotherapie lassen sich in distinkten Mustern von Geist wie Gehirn abbilden - Beispiel NET}

In einer randomisierten klinischen Studie [zur Veröffentlichung eingereicht] verglichen wir die Wirksamkeit der NET mit den Standardbehandlungen (TU; treatment as usual), die Traumatisierte in Deutschland erhalten. TUs konnten jede Art von Psychotherapie, auch in Kombination mit Pharmakotherapie, beinhalten, die derzeit Flüchtlingen in Deutschland angeboten wird. Überwiegend handelt es sich dabei um Stabilisierung und Ressourcenmobilisierung, aber auch um Entspannungsverfahren. Die NET folgte dem Manual von Schauer et al. [2005]. Da nur für die NET, aber nicht für die TUs eine theoretische Begründung und eine experimentelle Überprüfung [Neuner et al., 2004b; Onyut et al., 2005] vorlag, gingen wir von der Hypothese aus, dass die NET den TUs überlegen wäre und nur NET auch im Gehirn nachweisbare Veränderungen nach sich zöge. Die Katamnese schloss ein halbes Jahr ein. Erfasst wurden neben den klinischen Symptomen des Traumaspektrums über strukturierte Interviews auch die neurophysiologischen Rhythmen und Koppelungen mittels MEG und magnetischer Quellenlokalisation.

Die Untersuchung wurde an der nationalen «Psychologischen Forschungs- und Modellambulanz für Flüchtlinge» der Universität Konstanz durchgeführt, welche die Erforschung, Integration und Dissemination wissenschaftlicher Erkenntnisse der Epidemiologie, Diagnostik, Therapie und Menschenrechtsarbeit bei Überlebenden von Folter, Vergewaltigung, Krieg, Katastrophen und Terror zur Aufgabe hat. Aufgrund des Brückenschlags systematischer Forschungen einerseits im Labor und andererseits in Kriegs- und Krisengebieten werden konkrete psychodiagnostische und therapeutische Hilfestellungen in Kombination mit Menschenrechtsarbeit für Personen nach multipler Traumatisierung und Überlebende organisierter Gewalt erarbeitet und weiterentwickelt.

An der Untersuchung (November 2001 bis Oktober 2003) nahmen 32 Asylsuchende teil, die entweder auf Rat der behandelnden Therapeuten, von Richtern oder Anwälten oder auf eigenen Wunsch die Ambulanz in Konstanz aufgesucht hatten. In zufälliger Abfolge wurden Patienten der Ambulanz, die mit einer PTSD nach DSM-IV diagnostiziert worden waren, in eine der Behandlungsgruppen aufgenommen. Personen mit kognitiver Beeinträchtigung, neurologischen Erkrankungen, Psychosen oder Substanzabhängigkeit wurden nicht in die Untersuchung einbezogen. Nur 1 Patient nahm nach Aufklärung das Behandlungsangebot nicht an.

In beiden Gruppen waren jeweils 11 Männer und 5 Frauen. Das Alter betrug 31,1 \pm 7,8 Jahre in der NET- und 31,6 \pm 7,7 Jahre in der TU-Gruppe. Auch hinsichtlich anderer soziodemographischer Parameter (Schulbildung 7,3 Jahre, mittlere Anzahl der Kinder: 2,3) waren die beiden Gruppen vergleichbar. In beiden Gruppen überwiegten Asylsuchende kurdi- 
scher Ethnizität. Die Teilnehmer berichteten, im Mittel 4-5 verschiedene Arten traumatischer Erlebnisse erfahren $\mathrm{zu}$ haben, wobei fast bei allen Gefängnisaufenthalte dazugehörten und mehr als die Hälfte der Personen schwere Foltererlebnisse schilderte. Diese beinhalteten zwischen 5 und 40 verschiedene, systematische Foltertechniken (wie etwa Untertauchen in Wasser, Quetschen der Hoden, Aufhängen, sensorische Deprivation und andere).

Alle Untersuchungen wurden in Form strukturierter Interviews durchgeführt mit einem geschulten Übersetzer gleichen Geschlechts wie der Patient. Zum Zweck DSM-IV-basierter Diagnose, aber auch der Quantifizierung von Symptomschwere und -frequenz der PTSD diente das Composite International Diagnostic Interview [CIDI-K; WHO, 1997] sowie die Posttraumatic Stress Diagnostic Scale [PDS; Foa, 1995], als klinisches Interview durchgeführt. Zur Erfassung traumatischer Erfahrungen während Krieg, Gefangennahme und Folter diente die «vivo-Checklist of Organized Violence» (auf Anfrage von den Autoren erhältlich). Diese besteht aus 44 Items, die sich in die zwei Unterskalen «Foltererlebnisse» und «Kriegs- bzw. Kampferlebnisse» einteilen lassen.

Die Messung neuromagnetischer Hirnaktivität (MEG) erfolgte unter Ruhebedingen. Das MEG wurde systematisch und automatisiert auf abnorme Hirnaktivität in zwei Frequenzbändern (Delta und Theta) und ergänzend durch visuelle Inspektion untersucht. Extrahiert wurden fokal generierte Hirnwellen. Mittels t-Test wurden getrennt für beide Gruppen diejenigen Voxel identifiziert, in denen sich eine signifikante Veränderung von vor der Behandlung (prä) bis zum 6-MonatsFollow-up ergab.

Die NET umfasste im Mittel $9 \pm 4$ Behandlungssitzungen (Range 5-17, meist doppelstündige Sitzungen). Die Einschätzung des Therapeuten über die vollständige Bearbeitung traumatischer Erlebnisse und die diese begleitende Habituation bestimmten die Sitzungsanzahl. Die Sitzungen wurden wöchentlich oder auch zweimal die Woche anberaumt.

Für die Gesamtsumme der Symptomschwere belegte eine signifikante Wechselwirkung $(\mathrm{F}(1,28)=5,85 ; \mathrm{p}=0,02)$ den differentiellen Behandlungserfolg. Post-hoc-Tests zeigten eine signifikante Symptomreduktion in der NET-Gruppe $(\mathrm{p}<0,01)$, während in der TU-Gruppe keine überzufälligen Veränderungen $\mathrm{zu}$ beobachten waren (Abb. 1). Für die NET-Gruppe ergab sich eine Effektstärke von 1,6, für die TU-Gruppe nur von 0,4 . Schließlich zeigte sich auch bei Suizidalität eine signifikante Wechselwirkung mit $\mathrm{p}<0,05$ zugunsten der NETGruppe. Eine signifikante Veränderung der Medikation wurde in keiner der Gruppen beobachtet.

Die Veränderungen in den spontanen Rhythmen der Hirnaktivität sind in Abbildung 2 für einige axiale Hirnschnitte illustriert. In beiden Gruppen ergaben sich Voxel mit signifikanten Veränderungen. In allen entsprechenden Voxeln der NETGruppe änderte sich die Aktivität in Richtung einer «Normalisierung», d.h. in Richtung der Werte der Kontrollgruppe (Abb. 2, blau), während die überwiegende Zahl der Verände-

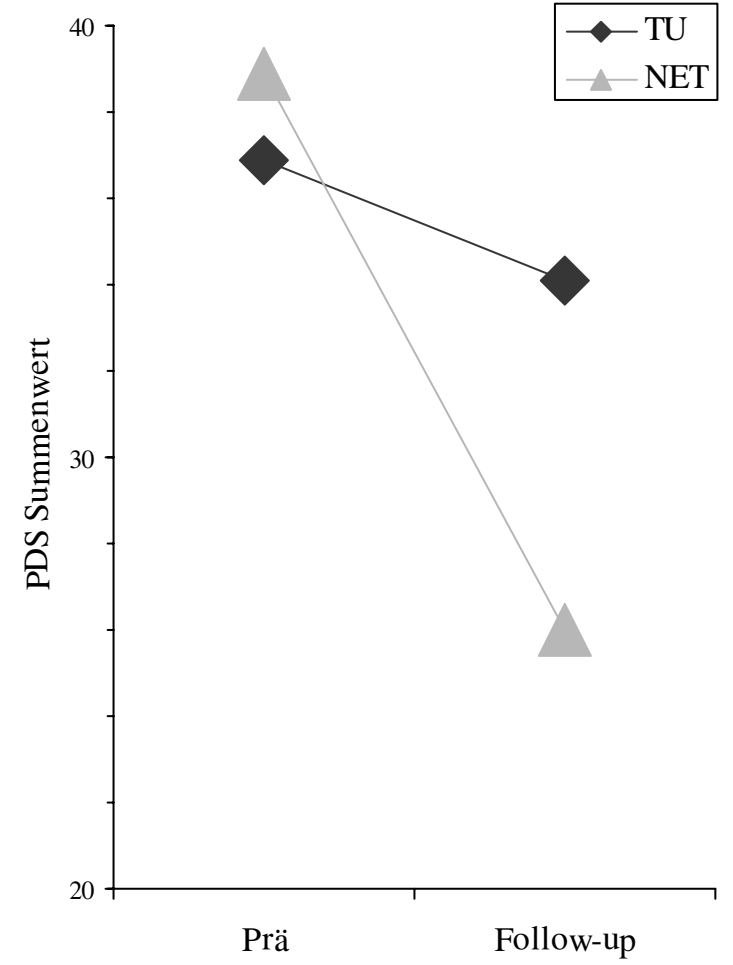

Abb. 1. Veränderung der mittleren Häufigkeit von PTSD-Symptomen über die Zeit; die NET-Gruppe, aber nicht die TU-Gruppe zeigt eine signifikante Reduktion im Behandlungsverlauf. Der PDS-Summenwert bestimmt sich aus der Summe der Symptome gewichtet mit 1-3, je nach Häufigkeit.

rungen in der TU-Gruppe auf zunehmend pathogenetische Aktivität hindeutete.

\section{Schlussfolgerung}

Im Folgenden wollen wir nur kurz die Befunde diskutieren, um dann etwas allgemeiner die Bedeutung neurophysiologischer Untersuchungen von psychotherapeutischen Prozessen zu erörtern.

Zunächst belegen die Ergebnisse, dass Symptome einer schweren PTSD auch dann signifikant gelindert werden können, wenn die Betroffenen wie hier einen unsicheren Aufenthaltsstatus haben, d.h. von Abschiebung bedroht sind und damit subjektiv und häufig auch objektiv unter akuter Bedrohung der Integrität von Leib und Leben stehen. Dies dürfte erklären, warum im Vergleich zu anderen Studien mit NET [Neuner et al., 2004b; Onyut et al., 2005; Schaal et al., 2006] der Erfolg der Symptomlinderung weniger ausgeprägt erscheint. Andererseits scheint die in Deutschland übliche Behandlung traumatisierter Asylsuchender diesen wenig Linderung zu bringen. In fast allen Fällen wurden Stabilisierung oder Entspannung, aber nie traumafokussierte Behandlungen angeboten, obwohl bis heute keine systematischen Studien 
Abb. 2. Dargestellt sind sechs axiale Tomogramme pro Gruppe, die von basal (links oben) schrittweise weitere dorsale Schnitte aufzeigen. Voxel mit signifikanten Veränderungen in den Hirnrhythmen zeigen unterschiedliche Prozesse in den beiden Gruppen an während in der TUGruppe abnormale langsame Wellen noch zunehmen (gelbe

Voxel), lässt sich in der NET-Gruppe im Therapieverlauf eine Annäherung der Hirnmuster an gesunde Kontrollen (signifikant in den blau markierten Voxeln) beobachten, ein Prozess der insbesondere im Frontallappen (hier prä- und orbitofrontal dargestellt) ausgeprägt ist.
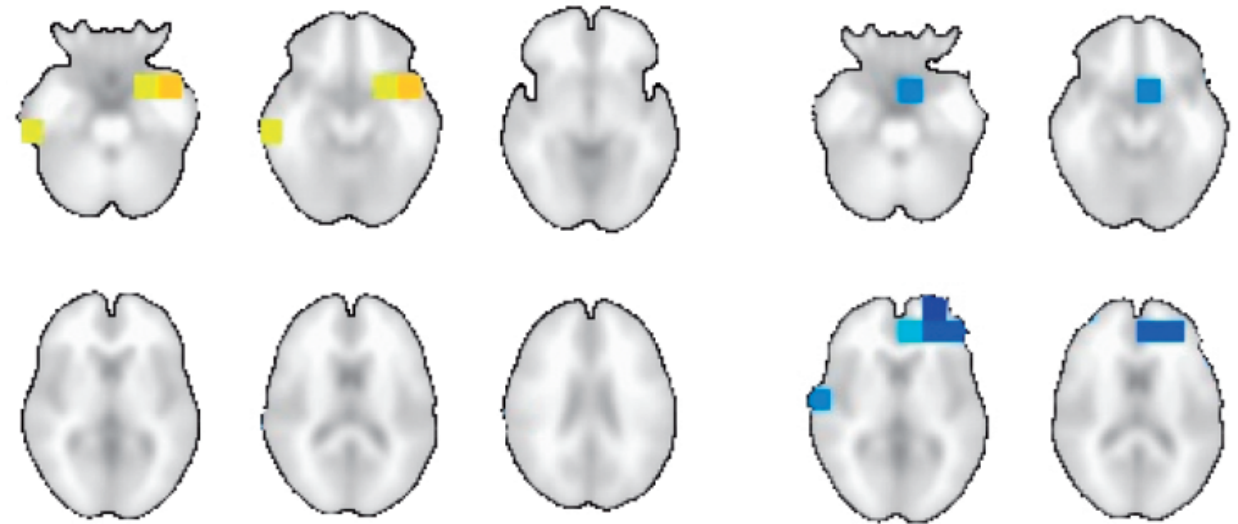
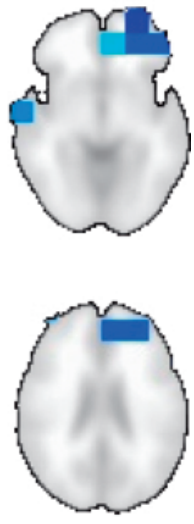

vorliegen, welche die Wirksamkeit von Stabilisierung belegen Ein Umdenken unter den behandelnden Therapeuten erscheint in dieser Hinsicht wünschenswert.

Wesentliche Veränderungen in der Hirnarchitektur mit Normalisierung in der Generatorstruktur abnormaler langsamer Hirnwellen waren nur in der NET-Gruppe zu beobachten. Aufgrund der Kontrolle der Medikation sowie der kontrollierten Anordnung der Untersuchung kann davon ausgegangen werden, dass die Hirnveränderungen durch die psychotherapeutische Intervention zustande gekommen sind. Dieses Phänomen ist bisher kaum untersucht worden. Bisherige Belege für wirksame Veränderungen von Verhaltenstherapie im Bereich der Hirnarchitektur konzentrierten sich eher auf den Bereich der Neurorehabilitation, wo (meist motorische) Übungen im Vordergrund stehen [z.B. Candia et al., 2004; Taub et al., 2002]. Der Nachweis, dass auch durch psychotherapeutische Behandlung, die sich auf Gespräche und Imaginationstechniken stützt, Veränderungen auf makroskopischer Ebene zu erzielen sind, ist daher keineswegs trivial. Dass wir aber eben diese Veränderungen mit nichtinvasiven Verfahren messen können, bietet uns einen neuen Zugang zum Verständnis psychotherapeutischer Prozesse und deren Weiterentwicklung. Im vorliegenden Fall lässt die beobachtete Normalisierung von Hirnfunktionen vermuten, dass das Furchtnetzwerk nicht mehr mit der früheren Intensität von anderen Bereichen entkoppelt wird und dass die «Entkopplung von Furcht und Sprache», die sich in langsamen Hirnwellen bemerkbar machen könnte [Ray et al., 2006], aufgehoben werden kann. Demnach würde NET also nicht einfach eine Hemmung der Furchtstrukturen bewirken, sondern eine Degradierung derart, dass nun andere (eben explizite) Informationen an die jeweiligen Elemente der Furchtstruktur angekoppelt werden. Dies bedingt dann möglicherweise über LTD eine Abschwächung der Furchtstruktur, deren einzelne Element verarbeitbar geworden sind. Mit zunehmender Zeit schwächt sich diese Struktur weiter, was die Langzeiterfolge von NET [Neuner et al., 2004b, Schaal et al., 2006] erklären könnte. In diesem Sinne würde NET den Betroffenen die Verarbeitung des traumatischen Geschehens und damit Hilfe zur Selbsthilfe ermöglichen. Daraus lassen sich testbare Hypothesen ableiten. Wir wollen aber an dieser Stelle weniger die Konsequenzen für erfolgreiche Traumatherapie aufzeigen, als vielmehr dieses Beispiel nutzen, um die Vorzüge therapiebegleitender neurophysiologischer Untersuchungen zusammenzufassen:

1. Physiologische Messungen können den Therapieerfolg validieren. Sie belegen relativ unangreifbar das Wirken (wenngleich nicht notwendigerweise die Wirksamkeit) entsprechender psychotherapeutischer Verfahren auch für den Nicht-Kliniker und sind damit in der gesellschaftlichen Diskussion hilfreich. Dies gilt natürlich insbesondere für die wenig versachlichte Diskussion im Bereich der Unterstützung von Flüchtlingen.

2. Physiologische Messungen können helfen, vermutete Wirkmechanismen einer Therapieform zu überprüfen, führen so zu besserer Modellierung und tragen damit zur Weiter- oder Neuentwicklung psychotherapeutischer Maßnahmen bei.

3. Klinische Psychologie und Psychiatrie, die sich oft auf Maße verlassen müssen, die eine an der Oberfläche sichtbare Endstrecke verschiedener Prozesse abbilden und damit unspezifisch geworden sind, können aus physiologischen Messungen tiefere und spezifischere Einsichten erwarten.

4. Die Kovarianz von Physiologie und Psychologie lehrt uns einmal mehr, dass Geist und Gehirn nur zwei Informationsebenen ein und desselben Phänomens darstellen und lassen uns damit vielleicht eines Tages die Trennung in eine Behandlung seelischer und körperlicher Erkrankungen überwinden. 


\section{Literatur}

Balliel B, Kissler J, Elbert T, Rockstroh B: «Hypersensibilität» für unangenehme Reize bei Patienten mit Posttraumatischer Belastungsstörung? Paper presented at the Jahrestagung der Deutschsprachigen Gesellschaft für Psychotraumatologie (DeGPT), Hannover, 2006.

Bao S, Chan VT, Merzenich MM: Cortical remodeling induced by activity of ventral tegmental dopamine neurons. Nature 2001;412:79-83.

Bao S, Chang EF, Davis JD, Gobeske KT, Merzenich MM: Progressive degradation and subsequent refinement of acoustic representations in the adult auditory cortex. J Neurosci 2003;23:10765-10775.

Candia V, Wienbruch C, Elbert T, Rockstroh B, Ray WJ: Effective behavioral treatment of focal hand dystonia in musicians alters somatosensory cortical organization. Proc Natl Acad Sci USA 2003;100:7942-7946. Charmandari E, Kino T, Souvatzoglou E, Chrousos GP: Pediatric stress: Hormonal mediators and human development. Horm Res 2003;59:161-179.

Elbert T, Bohnhoeffer T: Plastizität des Gehirns; in Deutsche Forschungsgemeinschaft (Hrsg): Perspektiven der Forschung und ihrer Förderung. Weinheim, Wiley-VCH, 2002, pp 287-300.

Elbert T, Rockstroh B: Reorganization of human cerebral cortex: the range of changes following use and injury. Neuroscientist 2004;10:129-141.

Elbert T, Pantev C, Wienbruch C, Rockstroh B, Taub $\mathrm{E}$ : Increased use of the left hand in string players associated with increased cortical representation of the fingers. Science 1995;270:305-307.

Elbert T, Rockstroh B, Kolassa I, Schauer M, Neuner $\mathrm{F}$ : The influence of organized violence and terror on brain and mind - a co-constructive perspective; in Baltes P, Reuter-Lorenz P, Rösler F (eds): Lifespan Development and the Brain: The Perspective of Biocultural Co-Constructivism. Cambridge University Press, 2006.

Foa E: PDS Posttraumatic Stress Diagnostic Scale. Minneapolis MN, NCS Pearson, 1995.

Gaser C, Schlaug G: Brain structures differ between musicians and non-musicians. J Neurosci 2003;23. 9240-9245.
Hellhammer D: Von Freud zu Meaney: Die Zukunft der Psychotherapie hat begonnen. Verhaltenstherapie 2005;15:76-77.

Iidaka T, Sadato N, Yamada H, Yonekura Y: Functional asymmetry of human prefrontal cortex in verbal and non-verbal episodic memory as revealed by fMRI. Cog Brain Res 2000;9:73-83.

Junghöfer M, Bradley M, Elbert T, Lang P: Fleeting images: A new look at early emotion discrimination. Psychophysiology 2001:38:175-178.

Junghöfer M, Schauer M, Neuner F, Odenwald M, Rockstroh B, Elbert T: Enhanced fear-network in torture survivors activated by RVSP of aversive material can be monitored by MEG. Psychophysiology 2003; 40(suppl):51.

Kilgard MP, Merzenich MM: Cortical map reorganization enabled by nucleus basalis activity. Science 1998; 279:1714-1718.

Kolassa I, Elbert T: Structural and functional changes in the brains of persons with Posttraumatic Stress Disorder (PTSD). Curr Dir Psychol Sci 2006, in press.

Lang P: A bio-informational theory of emotional imagery. Psychophysiology 1979;16:495-512.

Levine S: Infantile experience and resistance to physiological stress. Science 1957:126:405.

Levine S: Regulation of the hypothalamic-pituitaryadrenal axis in the neonatal rat: The role of maternal behavior. Neurotoxicity Res 2002;4:557-564.

Meaney JM: Maternal care, gene expression, and the transmission of individual difference in stress reactivity across generations. Ann Rev Neurosci 2001;24: 1161-1192.

Metcalve J, Jacobs W: A «hot-system/cool-system» view of memory under stress. PTSD Res Q 1996;7:1-3. Neisser U: Cognitive Psychology. Englewood Cliffs NJ, Prentice-Hall, 1967

Neuner F, Schauer M, Roth WT, Elbert T: Testimony therapy as an acute intervention in a macedonian refugee camp: Two case reports. Behav Cog Psychother 2002;30:205-209.
Neuner F, Schauer M, Karunakara U, Klaschik C, Robert C, Elbert T: Psychological trauma and evidence for enhanced vulnerability for PTSD through previous trauma in West Nile refugees. BMC Psychiatry 2004a; $4: 34$.

Neuner F, Schauer M, Klaschik C, Karunakara U, Elbert T: A comparison of narrative exposure therapy, supportive counseling, and psychoeducation for treating posttraumatic stress disorder in an african refugee settlement. J Consult Clin Psychol 2004b;72:579-587.

Onyut PL, Neuner F, Schauer E, Ertl V, Odenwald M, Schauer M, Elbert T: Narrative Exposure Therapy as a treatment for child war survivors with posttraumatic stress disorder: Two case reports and a pilot study in an African refugee settlement. BMC Psychiatry 2005;5:7. Ray WJ, Odenwald M, Neuner F, Schauer M, Ruf M, Rockstroh B, Elbert T: Decoupling neural networks from reality: Dissociative experiences in torture victims are reflected in abnormal brain waves in left frontal cortex. Psychol Sci 2006, in press.

Rockstroh B, Fehr T, Kissler J, Wienbruch C, Elbert T Magnetic source imaging of slow wave activity in psychiatric samples; in Nenonen J (ed): Biomag 2000. Helsinki, HUT, 2001, pp 395-398.

Schaal S, Elbert T: Ten years after the genocide. Trauma confrontation and PTSD in Rwandan adolescents. J Trauma Stress 2006;19:95-105.

Schauer M, Neuner F, Karunakara U, Klaschik C, Robert C, Elbert T: PTSD and the «building block» effect of psychological trauma among West Nile Africans. ESTSS Bull 2003;10:5-6.

Schauer M, Neuner F, Elbert T: Narrative Exposure Therapy. A short-term intervention for traumatic stress disorder after war, terror or torture. Göttingen, Hogrefe und Huber, 2005.

Steinberg L: Cognitive and affective development in adolescence. Trends Cog Sci 2005;9:69-74.

Taub E, Urswatte G, Elbert T: New treatments in neurorehabilitation founded on basic research. Nature Rev Neurosci 2002;3:228-236. 